\title{
The Analgesic Effects of Automatically Controlled Rotating Acupuncture in Rats: Mediation by Endogenous Opioid System
}

\author{
Sun Kwang KIM1, Hak Jin MooN11, Hyo Suk NA1, Kye Jin KIM11, Ji Hoon KIM1, \\ Jung Hyuk PARK1, Sang Hoon LEE2 ${ }^{2}$, Sung Soo RHIM³, Soon-Geul LEE³, and \\ Byung-II MIN 1,4 \\ 1Department of East-West Medicine, Graduate School, 2Department of Acupuncture \& Moxibustion, College of Oriental Medicine, \\ 3Department of Mechanical Engineering, College of Advanced Technology, and 4Department of Physiology, College of Medicine, \\ Kyung Hee University, Seoul, South Korea
}

\begin{abstract}
The technique of rotating acupuncture needles has long been used to enhance the effects of acupuncture in Oriental medicine. However, it is difficult to standardize and quantify this stimulation condition. Thus we developed an automatically controlled rotating acupuncture (ACRA) system. The present study was conducted to evaluate the analgesic effects of ACRA using 4 different stimulation conditions (i.e., angle and frequency of rotation: $90^{\circ}+1 \mathrm{~Hz}, 90^{\circ}+1 / 4 \mathrm{~Hz}, 360^{\circ}+1 \mathrm{~Hz}$, and $360^{\circ}+1 / 4 \mathrm{~Hz}$ ) in Sprague-Dawley rats. Tail-flick latency to a noxious radiant heat stimulus in lightly anesthetized rats was measured before
\end{abstract}

and after 15 min of ACRA stimulation at the Zusanli (ST36) acupoint. ACRA stimulations under all of the conditions above produced more potent analgesic effects than plain acupuncture (PA, acupuncture needle insertion only), but only the $90^{\circ}+1 / 4$ $\mathrm{Hz}$ ACRA condition showed a statistically significant effect versus PA $(P<0.01)$. Further, the analgesic effect of $90^{\circ}+1 / 4 \mathrm{~Hz}$ ACRA was reversed by pretreatment with naloxone $(2 \mathrm{mg} / \mathrm{kg}$, i.p.). These results indicate that the $90^{\circ}+1 / 4 \mathrm{~Hz}$ ACRA stimulation has the most potent analgesic effect in rats and that this is mediated by the endogenous opioid system.

Key words: acupuncture, rotation, opioid. \begin{abstract}
$\mathrm{A}_{\text {cupuncture has long been used in the Orient to treat }}$ various diseases. It has few side effects and is now viewed as an alternative method of medicine in Western countries $[7,11]$. Numerous studies have shown that acupuncture stimulation increases experimental pain thresholds in various animal species and man $[1,2,18]$. Traditionally, plain acupuncture (PA) uses manipulation (e.g., rotation of an acupuncture needle, varying its depth of insertion, or rapid needle insertion and withdrawal) to increase or complement its effects $[8,15,16]$. Rotation is among the most commonly used modes of acupuncture manipulation in clinical practice and in experiments $[4,6,8,12,14,18]$. However, it is difficult to standardize and quantify manually dependent stimulation conditions; thus animal studies on acupuncture with rotation were much smaller than on electroacupuncture (EA). We therefore developed an automatically controlled rotating acupuncture (ACRA) system to analyze the effect of stimulation conditions of rotation (i.e., the angle, frequency, amount and direction of rotation) in small animals, such as rats and mice, or in human subjects. The present study was performed to evaluate the analgesic effects of the devised ACRA system using different stimulation conditions and to investigate the involvement of the endogenous opioid system in ACRA-
\end{abstract}

induced analgesia.

Young adult male Sprague-Dawley rats (Sam: TacN[SD]BR, 200-250 g) were housed in groups of four with water and food available ad libitum. The room was maintained with a 12-h light/dark cycle (08:00-20:00 light, 20:00-08:00 dark) and kept at $23 \pm 2{ }^{\circ} \mathrm{C}$. The study was approved by the Institutional Animal Care and Use Committee of Kyung Hee University, and all procedures were conducted in accordance with the NIH guidelines.

The rats were lightly anesthetized with pentobarbital sodium $(40 \mathrm{mg} / \mathrm{kg}$, i.p.) to a level of anesthesia where tailflick reflexes were present, but no spontaneous movements or vocalization occurred $[8,16,17]$. Pain threshold was estimated by measuring tail-flick latency (TFL) elicited by a noxious radiant heat applied to the proximal third of the tail $[8,10,16,17]$. The intensity of the light bulb was set so that the baseline reaction time was $3.0 \pm 0.5 \mathrm{~s}$. When a TFL exceeded $7 \mathrm{~s}$ during an experimental procedure, the light bulb was switched off to avoid unnecessary skin damage. Fifteen min after anesthesia was administered, three successive TFL determinations were carried out at 1-min intervals, and these values were averaged and taken as basal TFL values. A stainless steel needle $(0.25$ $\mathrm{mm}$ in diameter and $30 \mathrm{~mm}$ long) was then inserted $(5 \mathrm{~mm}$ 
a
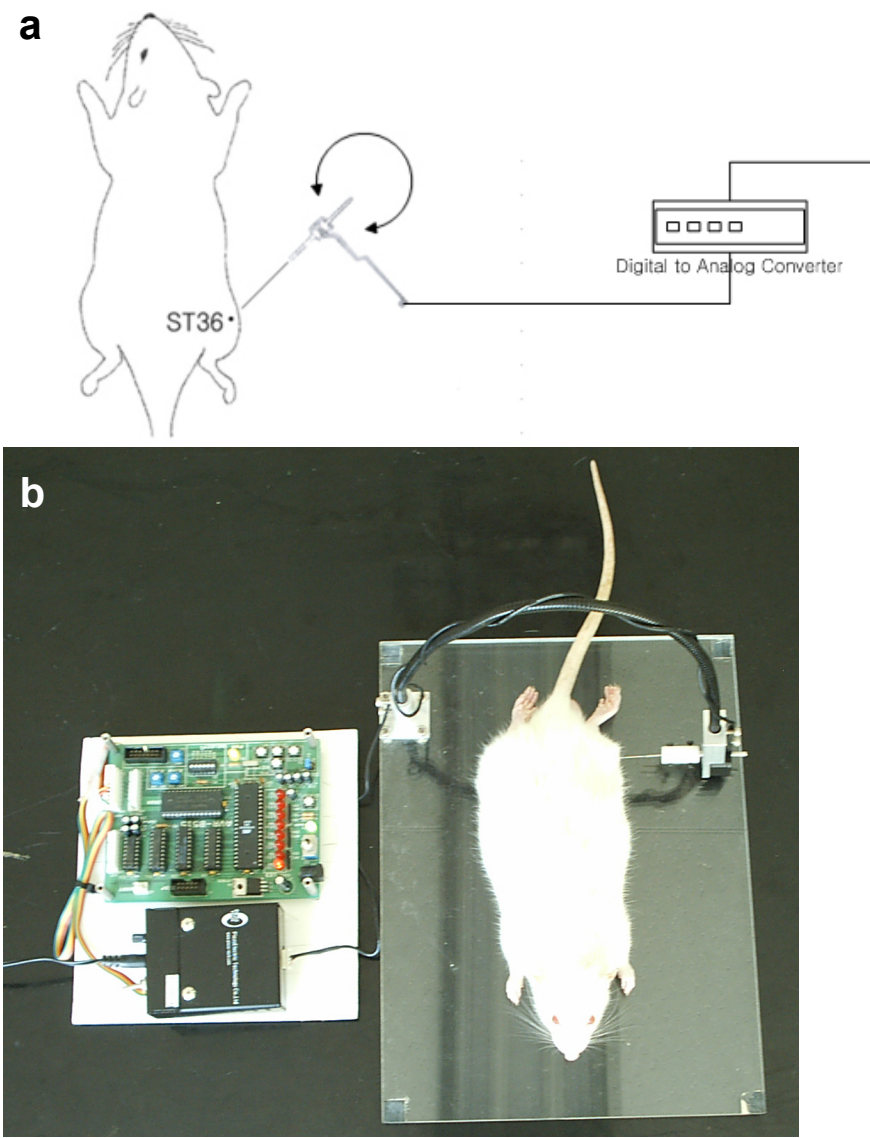

deep) in the Zusanli acupoint (ST 36), which is near the knee joint, $5 \mathrm{~mm}$ laterally and distally from the anterior tubercle of the tibia. Acupuncture stimulation at this point is known to produce an analgesic effect in the TFL test [8, 16]. The upper tip of the needle was mounted on the needle holder connected to the actuator (a cylindrical ultrasonic motor, $10 \mathrm{~mm}$ in diameter and $16 \mathrm{~mm}$ high, PiezoElectic Technology Co. Ltd., Korea) of the ACRA system. The AT90S8515 microcontroller controlled this actuator, which rotates the acupuncture needle. The operator can change frequencies and angles of rotation by using GUI (Graphic User Interface) commands from a personal computer interfaced with the microcontroller (Fig. 1). PA (acupuncture needle insertion only) and ACRA stimulations were performed for $15 \mathrm{~min}$. In the ACRA stimulations, acupuncture needles were rotated bidirectionally, with alternating clockwise and counterclockwise cycles, and four stimulation conditions (i.e., angle and frequency of rotation: $90^{\circ}+1 \mathrm{~Hz}, 90^{\circ}+1 / 4 \mathrm{~Hz}, 360^{\circ}+1 \mathrm{~Hz}$, and $360^{\circ}+1 / 4 \mathrm{~Hz}$ ) were examined. The average of three successive TFL determinations (postacupuncture TFL) was then recorded immediately after, and at 10, 20, and $30 \mathrm{~min}$ after the ACRA stimulation period. The analgesic effects are expressed as percent changes from basal TFL.

$$
\text { Acquired TFL change }=\frac{\text { postacupuncture TFL }- \text { basal TFL }}{\text { basal TFL }} \times 100 \%
$$

Fig. 1. The automatically controlled rotating acupuncture (ACRA) system: (a) schematic diagram of the ACRA system and (b) representative feature of the rat being stimulated by ACRA.

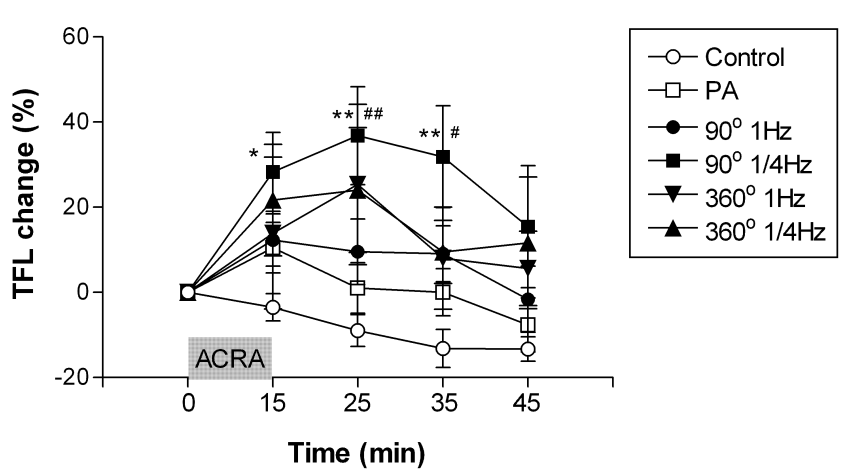

Fig. 2. Effects of ACRA stimulations on TFL by a noxious radiant heat stimulus in rats. Data are presented as means \pm SEM. $N=6$ rats/group. ${ }^{*} p<0.05,{ }^{* *} p<0.01$, vs. control group; $\# p<0.05, \# p<0.01$, vs. PA group by the NewmanKeuls multiple comparison test after one-way ANOVA. Control $=$ anesthesia only. PA = acupuncture needle insertion only.

In an additional set of experiments, naloxone $(2 \mathrm{mg} / \mathrm{kg}$, i.p.) or normal saline (sterile $0.9 \% \mathrm{NaCl}, 1 \mathrm{ml} / \mathrm{kg}$, i.p.) was injected $10 \mathrm{~min}$ after anesthesia was given. Five minutes later, basal TFL was measured, and ACRA stimulation was then performed for $15 \mathrm{~min}$. TFL was measured again $10 \mathrm{~min}$ after the stimulation was stopped. Based on the results of the first experiment, the most effective ACRA condition $\left(90^{\circ}+1 / 4 \mathrm{~Hz}\right)$ was applied. In all experiments, anesthetized rats that did not undergo acupuncture treatment were used as a control.

All data are presented as means \pm SEM. For statistical analysis, the one-way analysis of variance (ANOVA) followed by the Newman-Keuls multiple comparison test was used. In all cases, $P<0.05$ was considered significant.

The analgesic effects of ACRA stimulation are shown in Fig. 2. Of the 4 different stimulation conditions, $90^{\circ}+$ $1 / 4 \mathrm{~Hz}$ ACRA produced the greatest analgesic effect. After completing $15 \mathrm{~min}$ of stimulation, the $90^{\circ}+1 / 4 \mathrm{~Hz}$ ACRA group showed a statistically significant increase in TFL for up to $20 \mathrm{~min}$. The maximal analgesic effect of ACRA stimulation was observed 10 min after the $90^{\circ}+1 /$ $4 \mathrm{~Hz}$ ACRA stimulation was completed $(P<0.01$, vs. control or PA). Although ACRA stimulation using the other conditions (i.e., $90^{\circ}+1 \mathrm{~Hz}, 360^{\circ}+1 \mathrm{~Hz}, 360^{\circ}+1 / 4$ $\mathrm{Hz}$ ) also increased TFL more so than PA did, no statisti- 


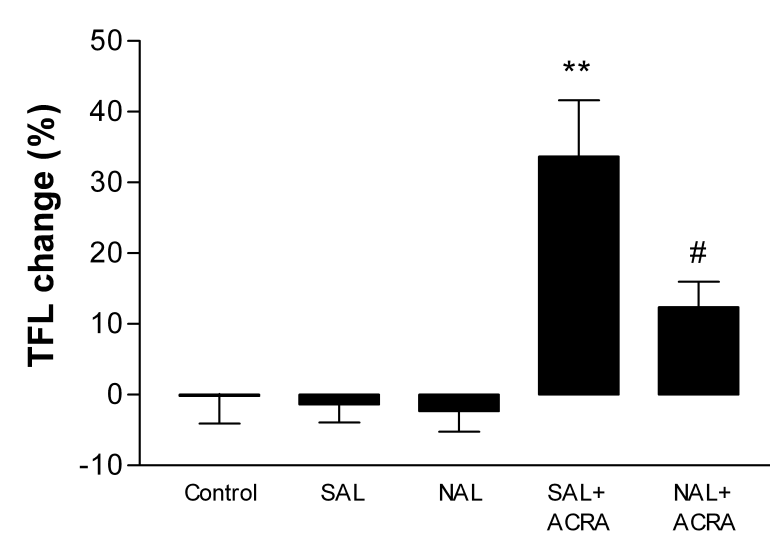

Fig. 3. Effect of naloxone pretreatment on the $90^{\circ}+1 / 4 \mathrm{~Hz}$ ACRA-induced analgesia. Data are presented as means \pm SEM. $N=6$ rats/group. ${ }^{* *} p<0.01$, vs. control group; $\# p<$ 0.05 , vs. SAL+ACRA group by Newman-Keuls multiple comparison test after one-way ANOVA. Control = anesthesia only. $\mathrm{SAL}=$ normal saline $(1 \mathrm{ml} / \mathrm{kg}$, i.p. $)$ only. $\mathrm{NAL}=$ naloxone $(2$ $\mathrm{mg} / \mathrm{kg}$, i.p.) only. SAL+ACRA = normal saline $(1 \mathrm{ml} / \mathrm{kg}$, i.p. $)$ pretreatment and $90^{\circ}+1 / 4 \mathrm{~Hz}$ ACRA stimulation. NAL+ $A C R A=$ naloxone $(2 \mathrm{mg} / \mathrm{kg}$, i.p. $)$ pretreatment and $90^{\circ}+1 / 4$ $\mathrm{Hz}$ ACRA stimulation.

cally significant difference was observed between groups $(P>0.05)$.

The results of naloxone pretreatment on $90^{\circ}+1 / 4 \mathrm{~Hz}$ ACRA-induced analgesia are shown in Fig. 3. The analgesic effect of $90^{\circ}+1 / 4 \mathrm{~Hz}$ ACRA stimulation was significantly blocked by naloxone pretreatment (NAL+ACRA group), but not by normal saline pretreatment (SAL+ ACRA group). Naloxone or normal saline alone did not affect basal TFL.

Since EA stimulation is well standardized and quantified, numerous studies have reported the analgesic effects of EA, using different stimulation conditions such as intensity, frequency, and duration of stimulation $[3,5,9$, 13]. However, the stimulation condition of rotating acupuncture needles is different, depending on the individual experimenter $[4,6,12,18]$, and few parametric studies of acupuncture with rotation have been performed. Therefore the technique requires standardization and quantification. In the present study, 4 different stimulation conditions were well controlled by use of the ACRA system.

The present study shows that the $90^{\circ}+1 / 4 \mathrm{~Hz}$ ACRA condition produced the greatest analgesic effect and that this effect was statistically significant when compared with the effect of PA. However, no significant TFL differences were found between the PA group and the other ACRA groups, though the other groups produced greater analgesic effects than PA did. The reasons for these results cannot be fully clarified at this time. Even though it is premature to define what the strong or weak stimulation of rotation is, we might consider rotating with a wider angle and a higher frequency as stronger stimulation, intuitively. In this regard, the results we made seem to suggest a cer- tain amount of moderate strength for the maximum effect of ACRA in terms of angle and frequency of rotation, rather than simply strong stimulation. In the second experiment of the present study, naloxone pretreatment was found to block the analgesic effect of $90^{\circ}+1 / 4 \mathrm{~Hz}$ ACRA stimulation, indicating that the endogenous opioid system, at least in part, mediates ACRA-induced analgesia. This corroborates the results from previous studies, showing the involvement of the opioid system in acupuncture analgesia [1-3]. However, the analgesic effect of $90^{\circ}+1 /$ $4 \mathrm{~Hz}$ ACRA stimulation was not completely blocked by naloxone, indicating that the non-opioid mechanism, such as the serotonergic and noradrenergic descending inhibitory system, also may be involved in the ACRA-induced analgesia $[1,2]$.

In conclusion, our findings suggest that the $90^{\circ}+1 / 4$ Hz ACRA stimulation has the strongest analgesic effect with respect to noxious heat stimuli in rats, and that this is mediated by the endogenous opioid system. Since EA with rotating acupuncture needles proved to have a greater analgesic effect than only EA did [8], we are currently developing a combined EA/ACRA system. Moreover, it would be of great interest to compare the analgesic effects of ACRA and other manipulation techniques.

This work was supported by a grant from the Ministry of Health \& Welfare, Republic of Korea (B050057).

\section{REFERENCES}

1. Filshie J, White A. Medical Acupuncture: a western scientific approach. Edinburg: Churchill Livingstone; 1998.

2. Han JS. The neurochemical basis of pain relief by acupuncture. Beijing: Chinese Medical Science and Technology Press; 1987.

3. Han JS. Acupuncture: neuropeptide release produced by electrical stimulation of different frequencies. Trends Neurosci. 2003;26:17-22.

4. Hsieh JC, Tu CH, Chen FP, Chen MC, Yeh TC, Cheng HC, Wu YT, Liu RS, Ho LT. Activation of the hypothalamus characterizes the acupuncture stimulation at the analgesic point in human: a positron emission tomography study. Neurosci Lett. 2001;307:105-8.

5. Huang C, Wang Y, Han JS, Wan Y. Characteristics of electroacupunctureinduced analgesia in mice: variation with strain, frequency, intensity and opioid involvement. Brain Res. 2002;945:20-5.

6. Kagitani F, Uchida S, Hotta H, Aikawa Y. Manual acupuncture needle stimulation of the rat hindlimb activates groups I, II, III and IV single afferent nerve fibers in the dorsal spinal roots. Jap J Physiol. 2005;55:149-55.

7. Kaptchuk TJ. Acupuncture: theory, efficacy, and practice. Ann Intern Med. 2002;136:374-83.

8. Kim JH, Min BI, Schmidt D, Lee HJ, Park DS. The difference between electroacupuncture $(E A)$ only and $E A$ with manipulation on analgesia in rats. Neurosci Lett. 2000;279:149-52.

9. Lao L, Zhang RX, Zhang G, Wang X, Berman BM, Ren K. A parametric study of electroacupuncture on persistent hyperalgesia and Fos protein expression in rats. Brain Res. 2004;1020:18-29.

10. Le Bars D, Gozariu M, Cadden SW. Animal models of nociception. Pharmacol Rev. 2001:53:597-652.

11. NIH consensus conference. Acupuncture. JAMA. 1998;280:1518-24.

12. Pohodenko-Chudakova $I O$. Acupuncture analgesia and its application in craniomaxillofacial surgical procedures. J Craniomaxillofac Surg. 2005;33:118-22.

13. Romita VV, Suk A, Henly JL. Parametric studies on electroacupuncture-like stimulation in a rat model: Effects of intensity, frequency, and duration of stimulation on evoked antinociception. Brain Res Bull. 1997;42:289-96.

14. Sumiya E, Kawakita K. Inhibitory effects of acupuncture manipulation and focal electrical stimulation of the nucleus submedius on a viscerosomatic reflex in 


\section{S.K. Kim et al.}

anesthetized rats. Jpn J Physiol. 1997;47:121-30.

15. Takakura N, Ogawa H, lijima S. Nishimura K, Kanamaru A, Sibuya M, Homma I, Effect of acupuncture at the Hoku point on vibration-induced finger flexion reflex in man: comparison between press needle technique, electroacupuncture, and in-situ technique. Am J Chin Med. 1995;23:313-8.

16. Uom ES, Min BI, Kim JH, Cho YW. Analgesic effect of the acupuncture using the method of quick insertion and withdrawal of the needle in rats. Neurosci Lett.
2001;298:21-4.

17. Wang $Q$, Mao LM, Han JS. Characterization of inhibition of spinal nociceptive reflex by stimulation of the arcuate nucleus of the hypothalamus in the pentobarbital-anesthetized rats. Pain. 1990;41:101-18.

18. Zaslawski CJ, Cobbin D, Lidums E, Petocz P. The impact of site specificity and needle manipulation on changes to pain pressure threshold following manual acupuncture: a controlled study. Complement Ther Med. 2003;11:11-21. 